Portland State University

PDXScholar

University Honors Theses

University Honors College

Spring 2021

\title{
Spanning Trees of Complete Graphs and Cycles
}

Minjin Enkhjargal

Portland State University

Follow this and additional works at: https://pdxscholar.library.pdx.edu/honorstheses

Part of the Other Mathematics Commons

Let us know how access to this document benefits you.

Recommended Citation

Enkhjargal, Minjin, "Spanning Trees of Complete Graphs and Cycles" (2021). University Honors Theses.

Paper 1120.

https://doi.org/10.15760/honors.1151

This Thesis is brought to you for free and open access. It has been accepted for inclusion in University Honors Theses by an authorized administrator of PDXScholar. Please contact us if we can make this document more accessible: pdxscholar@pdx.edu. 


\section{Spanning Trees of Complete Graphs and Cycles}

by

\section{Minjin Enkhjargal}

An undergraduate honors thesis submitted in partial fulfillment of the requirements for the degree of

Bachelor of Science

in

University Honors

and

Mathematics

Thesis Adviser

John Caughman

Portland State University 


\begin{abstract}
Spanning trees are typically used to solve least path problems, and several algorithms exist for finding the minimal spanning tree of a graph. Given a number $\mathrm{t} \geq 3$ what is the least number $\mathrm{n}=\alpha(\mathrm{t})$ such that there exists a graph on $n$ vertices having precisely $t$ spanning trees? In this work, we consider how the factoring of $t$ and the use of cycles connected by one vertex will affect $\alpha(\mathrm{t})$ ? Lower and upper bounds of $\alpha(\mathrm{t})$ are graphed to illustrate bounds using properties of cycles and complete graphs. The upper bound of $\alpha(\mathrm{t})$ is then improved by constructing a graph of connected cycles $\left\{\mathrm{C}_{\mathrm{p} 1}, \mathrm{C}_{\mathrm{p} 2}\right.$, $\left.\mathrm{C}_{\mathrm{p} 3}, \ldots, \mathrm{C}_{\mathrm{pn}}\right\}$ where $\mathrm{p} 1, \mathrm{p} 2, \mathrm{p} 3 \ldots \mathrm{pn}$ belong to the prime factorization of $\mathrm{t}$. The bounds of $\alpha(\mathrm{t})$ are significantly improved.
\end{abstract}

\title{
1 Introduction
}

Graph theory is one of the many areas of study that has grown popular in modern mathematics. Ideas that originated from graph theory are used in applications of mathematics and in other areas of study as well. Graphs are structures that consist of vertices and edges. One property of graphs that is often studied is its number of spanning trees. A spanning tree of a graph is a subgraph that contains the least number of edges needed to create a path from one vertex to another until all vertices are connected [1].

The discussion of least spanning trees or the more commonly discussed problem of finding the shortest path is popular among researchers in computer science and computer networking. It is an important theoretical foundation for many algorithms used in graph data structures in computer science, civil network planning, and computer network routing. [2] These applications use minimal spanning trees to find the least path or most efficient subgraph containing no cycles, which can provide information on finding the most efficient and economical solution. In many modern technologies, efficiency is a crucial demand for applications using mathematics and computer science.

A function related to spanning trees of graphs is $\alpha(t)=n$, which takes in the desired number of spanning trees of some graph $G$, denoted $t=\tau(G)$, as the parameter. The output $n$ equals the vertices of graph $G$, where $G$ contains the least number of vertices of any graph that has exactly t spanning trees. In 1970, graph theorist J. Sedlacek [3] proved some properties about the asymptotic behavior of $\alpha(t)$. He showed 
that $\alpha(\mathrm{t}) \leq \frac{t+6}{3}$ if $\mathrm{t} \equiv 0(\bmod 3)$ and $\alpha(\mathrm{t}) \leq \frac{t+4}{3}$ if $\mathrm{t} \equiv 2(\bmod 3)$. Later, the known upper bound of $\alpha(t)$ is improved by J. Azarija and R. Skrekovski. They prove [4] that $\alpha(\mathrm{t}) \leq \frac{t+9}{4}$ and $\beta(\mathrm{t}) \leq \frac{t+13}{4}$, if $\mathrm{t} \notin\{3,4,5,6,7,9,10,13,18,22\}$. They also show that the known fixed points of $\alpha$ are 3, 4, 5, 6, 7, 9, 10, 13, 18, 22, which is the subset of Euler's Idoneal numbers. Additionally, Azarija and Skrekovski also consider $\beta(\mathrm{t})=\mathrm{k}$, the analogous function minimizing edges of graphs with $t$ trees. A formula for the optimal values of these functions has not been found yet. A conjecture has been made [5] in the math community that $\alpha(n)=\mathrm{o}(\log \mathrm{n})$.

We now fix our notation and summarize the basic question of this paper. Given a number $\mathrm{t} \geq 3$ what is the least number $\mathrm{n}=\alpha(\mathrm{t})$ such that there exists a graph on $\mathrm{n}$ vertices having precisely $\mathrm{t}$ spanning trees? Let there exist a graph $\mathrm{G}$ with $t$ spanning trees. Then $\alpha(t)$ is the order of any graph with the least number of vertices of such a graph.

\subsection{Definitions}

Graph: A graph is a mathematical representation of a network. It represents the relationship between lines and points. A graph is given as $G=(V, E)$, where $V$ is the set of vertices and $\mathrm{E}$ is the set of edges.

Spanning Tree: A spanning tree is a subgraph of any graph that contains the least number of edges needed to create a path, with no cycles, from one vertex to another until all vertices are connected. All spanning trees have (n-1) edges, for a graph with $n$ vertices.

Complete Graph: A complete graph is a simple undirected graph with each pair of distinct vertices connected by a unique edge. The complete graph on $\mathrm{n}$ vertices is denoted by $\mathrm{K}_{\mathrm{n}}$.

Cycle: A cycle is a simple graph with $n$ vertices, where $n>2$, with edges connecting each vertex in a cyclic order of length $n$. The cycle on $n$ vertices is denoted by $C_{n}$.

\subsection{Spanning Tree Counting Theorems}

There are two common techniques for counting spanning trees. Let $\mathrm{G}$ be any graph, then $\tau(G)$ outputs the number of spanning trees for graph $G$. 


\section{Deletion-Contraction Formula}

This technique uses a deletion-contraction formula in a recursive form to count spanning trees of a graph. For a graph $\mathrm{G}, \tau(\mathrm{G})=\tau(\mathrm{H}-\mathrm{e})+\tau(\mathrm{H} \bullet \mathrm{e})$.

\section{Kirchhoff's Matrix-Tree Theorem}

This technique uses the adjacency matrix of a graph to create the Laplacian matrix denoted L. With matrix L, a row and column is crossed off to get the smaller matrix L*. Then, for a given graph $G, \tau(G)=\operatorname{det}\left(L^{*}\right)[6]$.

\section{Cayley's Tree Formula}

Cayley's tree formula [7] is based off the matrix-tree theorem. Let $T_{n, k}$ be the number of labelled forests on $\mathrm{n}$ vertices with $\mathrm{k}$ connected components, then $\mathrm{T}_{\mathrm{n}, \mathrm{k}}=\mathrm{kn}^{\mathrm{n}-\mathrm{k}-1}$. For the spanning trees of complete graphs, $\tau\left(K_{n}\right)=n^{(n-2)}$.

\section{Bounds of $\alpha(\mathrm{n})$ using cycles and complete graphs}

In a related discussion of this topic, the spanning trees of circulant graphs are also worth mentioning. The spanning trees of this subclass of regular graphs correlates closer to the applications of computer network routing. A circulant graph is defined [8] as "a graph of $\mathrm{n}$ graph vertices in which the $i$-th graph vertex is adjacent to the $(i+j)$-th and $(i-j)$-th graph vertices for each $j$ in a list l." When visualized, these graphs are seen to be formed from cycles, but with additional adjacencies.

1. The spanning trees of the circulant graph, square of the cycle $\mathrm{C}^{1,2}{ }_{\mathrm{n}}[9]$, is $\tau\left(\mathrm{C}^{1,2}{ }_{\mathrm{n}}\right)$ $=\mathrm{nF}^{2}{ }_{\mathrm{n}}$, where $\mathrm{F}_{\mathrm{n}}$ is the sequence of the Fibonacci numbers.

\section{Fibonacci}

$\begin{array}{ccc}\mathrm{N} & \text { Sequence } & \tau\left(\mathrm{C}^{1,2} \mathrm{n}\right) \\ 1 & 1 & 1 \\ 2 & 1 & 2 \\ 3 & 2 & 12 \\ 4 & 3 & 36 \\ 5 & 5 & 125 \\ 6 & 8 & 384 \\ 7 & 13 & 1183 \\ 8 & 21 & 3528 \\ 9 & 34 & 10404 \\ 10 & 55 & 30250\end{array}$


2. The number of spanning trees for complete graphs is $\tau\left(K_{n}\right)=n^{(n-2)}$

$\begin{array}{cc}\mathbf{n} & \tau\left(\mathrm{K}_{\mathrm{n}}\right) \\ 1 & 1 \\ 2 & 1 \\ 3 & 3 \\ 4 & 16 \\ 5 & 125 \\ 6 & 1296 \\ 7 & 16807 \\ 8 & 262144 \\ 9 & 4782969 \\ 10 & 100000000\end{array}$

\subsection{Fixed Points of $\alpha(\mathrm{t})$}

By Azarija and Skrekovski, $\alpha(t)$ is fixed for the subset of Euler's Idoneal numbers: $\{3,4$, $5,6,7,9,10,13,18,22\}$ For this set, the $\alpha(\mathrm{t})$ cannot be reduced and is fixed.

\begin{tabular}{|cc|}
\hline $\mathrm{n}$ & $\alpha(\mathrm{t})=\mathrm{n}$ \\
\hline 3 & 3 \\
4 & 4 \\
5 & 5 \\
6 & 6 \\
7 & 7 \\
9 & 9 \\
10 & 10 \\
13 & 13 \\
18 & 18 \\
22 & 22 \\
\hline
\end{tabular}

\subsection{Upper Bounds of $\alpha(\mathrm{t})$}

Upper Bound for a Cycle: A cycle with $n$ vertices has exactly $n$ spanning trees. Each spanning tree of a cycle is found by removing a single edge to produce a unique spanning tree. Then the maximum upper bound of $\alpha(t)$ for the set of cycles $C_{n}$ is $\tau\left(C_{n}\right)$ $=\mathrm{n}$. Therefore, $\alpha\left(\tau\left(\mathrm{C}_{\mathrm{n}}\right)\right) \leq \mathrm{n}$. 


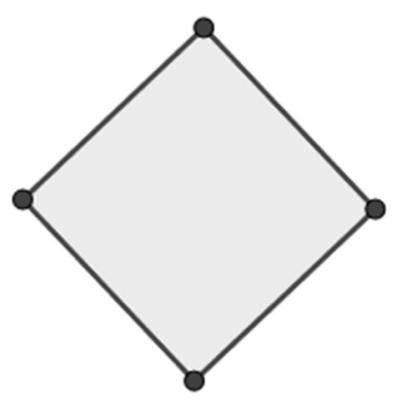

$\mathrm{n}=4$

$\tau\left(\mathrm{C}_{4}\right)=4$

$\alpha(4) \leq 4$

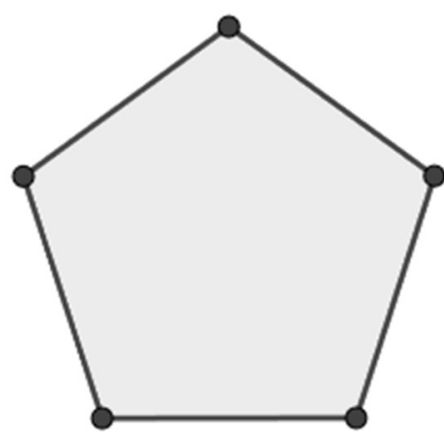

$\mathrm{n}=5$

$\tau\left(\mathrm{C}_{5}\right)=5$

$\alpha(5) \leq 5$

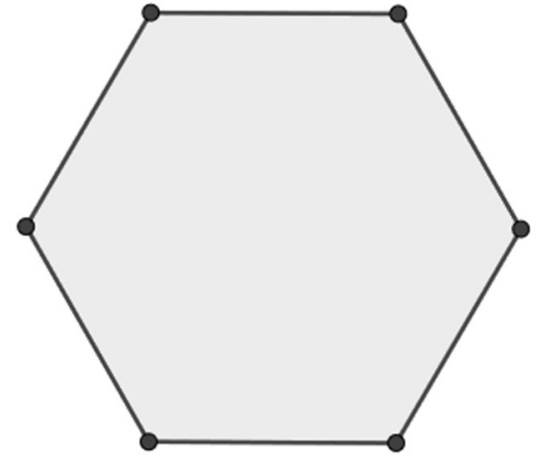

$\mathrm{n}=6$

$\tau\left(\mathrm{C}_{6}\right)=6$

$\alpha(6) \leq 6$

Figure 1: For cycles $\mathrm{C}_{4}, \mathrm{C}_{5}$, and $\mathrm{C}_{6}$ the number of spanning trees is equal to the number of vertices of that cycle and $\alpha\left(\tau\left(\mathrm{C}_{n}\right)\right)$ is less than or equal to $\mathrm{n}$.

Upper bound for complete graphs: The set of complete graphs denoted by $\mathrm{K}_{\mathrm{n}}$ is one that contains edges from each vertex to another. For any given cycle with $n$ vertices $>4$, adding additional edges between vertices will allow the graph to produce a greater number of spanning trees. Then a complete graph $\mathrm{K}_{\mathrm{n}}$ will produce the most spanning trees for $n$ vertices, since $\mathrm{K}_{\mathrm{n}}$ contains edges from every vertex to all other vertices. Therefore $\alpha\left(\tau\left(K_{n}\right)\right) \leq n$.

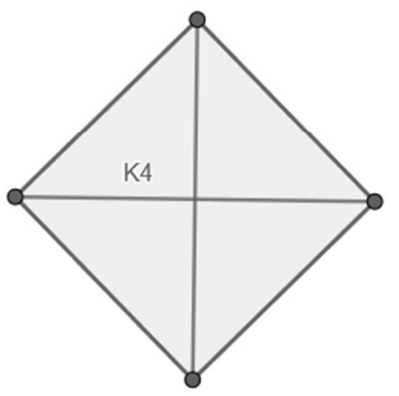

$\mathrm{n}=4$

$\tau\left(\mathrm{K}_{4}\right)=16$

$\alpha(16) \leq 4$

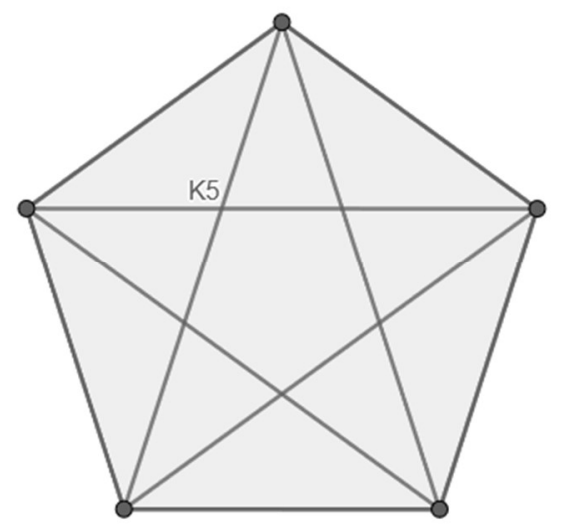

$\mathrm{n}=5$

$\tau\left(\mathrm{K}_{5}\right)=125$

$\alpha(125) \leq 5$

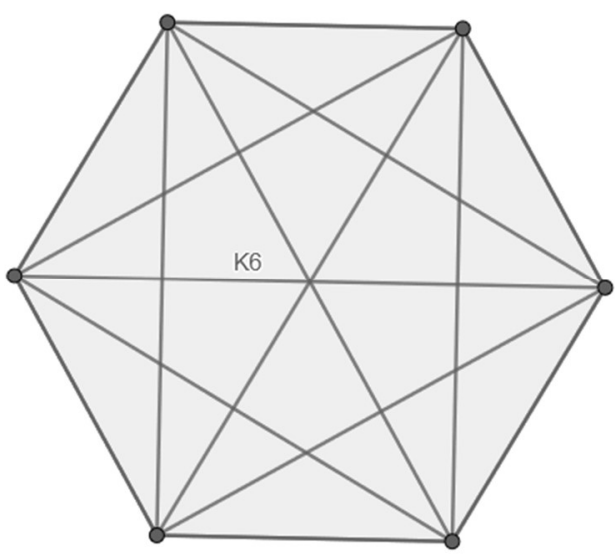

$\mathrm{n}=6$

$\tau\left(\mathrm{K}_{6}\right)=1296$

$\alpha(1296) \leq 6$

Figure 2: For complete graphs $\mathrm{K}_{4}, \mathrm{~K}_{5}$, and $\mathrm{K}_{6}$ the number of spanning trees is equal to the number of vertices of that cycle and $\alpha\left(\tau\left(K_{n}\right)\right)$ is less than or equal to n. $\tau\left(K_{4}\right)$ is higher than that of $C_{n}$ since it contains more edges. 
For each $n, \mathrm{C}_{\mathrm{n}}$ and $\mathrm{K}_{\mathrm{n}}$ represent the cycles and complete graphs, respectively, with $\mathrm{n}$ vertices. Let $t=\tau\left(K_{n}\right)$. Then taking $\alpha\left(\tau\left(K_{n}\right)\right)$ allows us to determine some upper and lower bounds for $\alpha(\mathrm{t})$. Clearly, the maximum upper bound is $\alpha(\mathrm{t}) \leq \mathrm{t}$ so, $\alpha\left(\tau\left(\mathrm{K}_{\mathrm{n}}\right)\right) \leq$ $\tau\left(\mathrm{K}_{\mathrm{n}}\right)$. The lower bound is determined by the result of $\alpha\left(\tau\left(\mathrm{K}_{\mathrm{n}}\right)\right)$.

Let $\mathrm{n}=4$. Note that: $\tau\left(\mathrm{K}_{4}\right)=16$ and $\alpha(16) \leq 4$

Any graph with $\mathrm{n}=4$ vertices can have at most $\tau\left(\mathrm{K}_{4}\right)=16$ spanning trees.

For any graph with $16+1$ spanning trees, since $\mathrm{n}=4$ has at most 16 spanning trees, $\alpha(17)$ must be a graph with 5 or more vertices.

$$
5 \leq \alpha(17)
$$

Then it is true that

$$
4<\alpha(17)
$$

It is also true that

$4 \leq \alpha(16)$

Then the bounds are

$$
\mathrm{n} \leq \alpha\left(\tau\left(\mathrm{K}_{\mathrm{n}}\right)\right) \leq \tau\left(\mathrm{K}_{\mathrm{n}}\right)
$$

\begin{tabular}{|cccc|}
\hline $\mathrm{n}$ & $\tau\left(\mathrm{C}_{\mathrm{n}}\right)$ & $\tau\left(\mathrm{K}_{\mathrm{n}}\right)$ & Bounds of $\alpha$ \\
\hline 3 & 3 & 3 & $3 \leq \alpha(3) \leq 3$ \\
4 & 4 & 16 & $4 \leq \alpha(16) \leq 16$ \\
5 & 5 & 125 & $5 \leq \alpha(125) \leq 125$ \\
6 & 6 & 1296 & $6 \leq \alpha(1296) \leq 1296$ \\
7 & 7 & 16807 & $7 \leq \alpha(16807) \leq 16807$ \\
8 & 8 & 262144 & $8 \leq \alpha(262144) \leq 262144$ \\
9 & 9 & 4782969 & $10 \leq \alpha(100000000) \leq 100000000$ \\
10 & 10 & 100000000 & $12 \leq \alpha(2357947691) \leq 2357947691$ \\
11 & 11 & 2357947691 & $13 \leq \alpha(179216039394037) \leq 1792160394037$ \\
12 & 12 & 61917364224 & $14 \leq \alpha(56693912375296) \leq 56693912375296$ \\
13 & 13 & $1.79216 \mathrm{E}+12$ & $15 \leq \alpha(1946195068359370) \leq 1946195068359370$ \\
14 & 14 & $5.66939 \mathrm{E}+13$ & \\
15 & 15 & $1.9462 \mathrm{E}+15$ &
\end{tabular}

These upper bounds can be improved by taking the prime factorization of the upper bound. Note that the upper bound is the result of the number of spanning trees for that cycle (i.e. $\left.\mathrm{C}_{16807}=>\tau\left(\mathrm{C}_{16807}\right)=16807\right)$ Therefore, taking the prime factorization of 
16807, a graph of connected cycles can be constructed, where each sub-cycle contains the same number of vertices as each of the primes in the prime factorization.

Let $\mathrm{G}$ be the cycle $\mathrm{C}_{\mathrm{t}}$. Let $\mathrm{H}$ be the graph constructed of cycles from the set $\left\{\mathrm{C}_{\mathrm{p} 1}, \mathrm{C}_{\mathrm{p} 2}\right.$, $\mathrm{C}_{\mathrm{p} 3}, \ldots, \mathrm{C}_{\mathrm{pn}} \mid \mathrm{p} 1, \mathrm{p} 2, \mathrm{p} 3, \ldots, \mathrm{pn} \in$ prime factorization of $\left.\mathrm{t}\right\}$. If the prime factorization includes any powers of 2 , it must be converted to a power of 4 , since there cannot be a cycle of 2 vertices, without requiring duplicate edges.

\begin{tabular}{|cc|}
\hline $\mathrm{t}$ & Prime Factorization of t \\
\hline 16 & $4 \times 4$ \\
125 & $5 \times 5$ \\
1296 & $4 \times 4 \times 3 \times 3 \times 3 \times 3$ \\
16807 & $7 \times 7 \times 7 \times 7 \times 7$ \\
262144 & $4 \times 4 \times 4 \times 4 \times 4 \times 4 \times 4 \times 4 \times 4$ \\
4782969 & $3 \times 3 \times 3 \times 3 \times 3 \times 3 \times 3 \times 3 \times 3 \times 3 \times 3 \times 3 \times 3 \times 3$ \\
100000000 & $4 \times 4 \times 4 \times 4 \times 5 \times 5 \times 5 \times 5 \times 5 \times 5 \times 5 \times 5$ \\
2357947691 & $11 \times 11 \times 11 \times 11 \times 11 \times 11 \times 11 \times 11 \times 11$ \\
61917364224 & $4 \times 4 \times 4 \times 4 \times 4 \times 4 \times 4 \times 4 \times 4 \times 4 \times 3 \times 3 \times 3 \times 3 \times 3 \times 3 \times 3 \times 3 \times 3 \times 3$ \\
1792160394037 & $13 \times 13 \times 13 \times 13 \times 13 \times 13 \times 13 \times 13 \times 13 \times 13 \times 13$ \\
\hline
\end{tabular}

\section{Example.}

Let $\mathrm{G}=\mathrm{C}_{1296}$. The prime factorization of $1296=2^{4 *} 3^{4}=4 \times 4 \times 3 \times 3 \times 3 \times 3$.

Let $\mathrm{H}$ be the graph constructed of cycles $\mathrm{C}_{4}, \mathrm{C}_{4}, \mathrm{C}_{3}, \mathrm{C}_{3}, \mathrm{C}_{3}, \mathrm{C}_{3}$. Then $\mathrm{H}$ is such a graph:

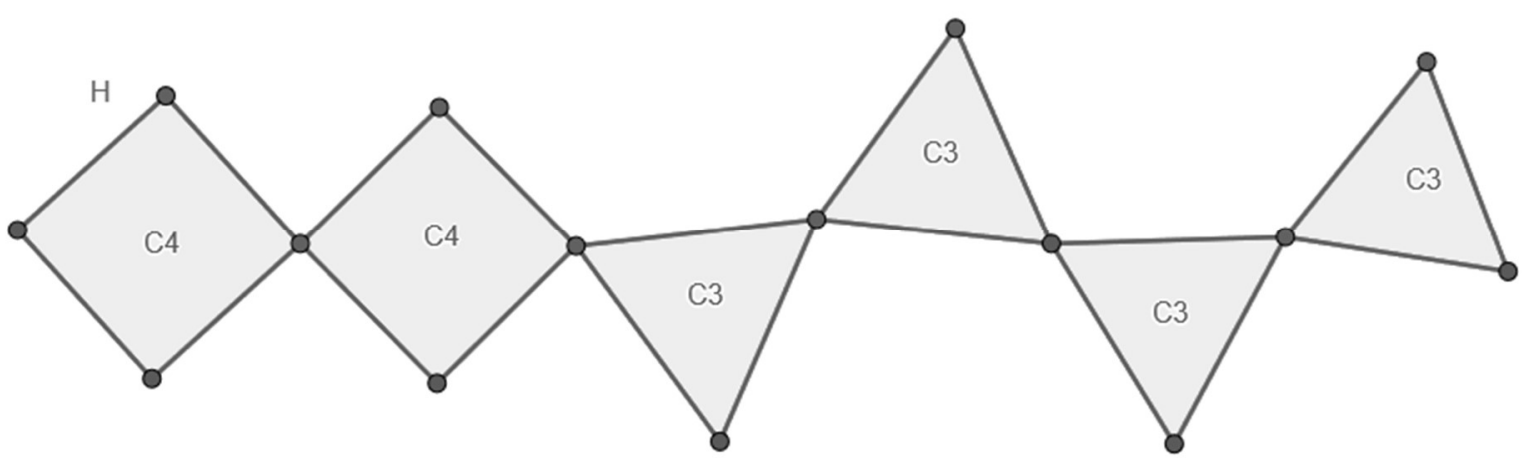

$\mathrm{H}$ contains the same number of spanning trees as $\mathrm{G}$. To see this, choose one spanning tree from each sub-cycle in $\mathrm{H}$. The combined outcome is one spanning tree of $\mathrm{H}$. To find the second spanning tree of $\mathrm{H}$, the chosen spanning trees for the sub-cycles shall remain the same. Now choose a different spanning tree for only the first sub-cycle $\mathrm{C}_{4}$. To find 
the third spanning tree of $\mathrm{H}$, choose a different spanning tree of $\mathrm{C}_{4}$. This can be repeated for each sub-cycle. $\mathrm{C}_{4}$ has 4 choices of spanning trees and $\mathrm{C}_{3}$ has 3 choices of spanning trees. Then this becomes a counting problem where the total number of spanning trees is equal to $4 \times 4 \times 3 \times 3 \times 3 \times 3$, which is the same as the factorization of 1296.

Thus, $\mathrm{G}=\mathrm{C}_{\mathrm{t}}$ and $\mathrm{H}$ both have the same number of spanning trees. While $\mathrm{C}_{\mathrm{t}}$ has $\mathrm{t}$ vertices, $\mathrm{H}$ has less vertices since each sub-cycle shares a vertex. The number of vertices for a set of sub-cycles $\left\{\mathrm{C}_{\mathrm{p} 1}, \mathrm{C}_{\mathrm{p} 2}, \mathrm{C}_{\mathrm{p} 3}, \ldots, \mathrm{C}_{\mathrm{pn}}\right\}$ is calculated by taking the sum $\mathrm{p}_{1}+\mathrm{p}_{2}+$ $\mathrm{p}_{3}+\ldots+\mathrm{p}_{\mathrm{n}}-\mathrm{n}+1$ (the number of sub-cycles in the graph). For graph $\mathrm{H}$, the number of vertices is $4+4+3+3+3+3-5=15$. Then there now exists a graph with significantly less vertices than the original cycle. The updated bounds for this set of graphs is:

\begin{tabular}{|ccc|}
\hline $\mathrm{t}$ & Vertices & Bounds of $\alpha$ \\
\hline 16 & 7 & $4 \leq \alpha(16) \leq 7$ \\
125 & 9 & $5 \leq \alpha(125) \leq 9$ \\
1296 & 15 & $6 \leq \alpha(1296) \leq 15$ \\
16807 & 31 & $7 \leq \alpha(16806) \leq 31$ \\
262144 & 28 & $8 \leq \alpha(262144) \leq 28$ \\
4782969 & 29 & $9 \leq \alpha(4782969) \leq 29$ \\
100000000 & 45 & $10 \leq \alpha(100000000) \leq 45$ \\
2357947691 & 91 & $11 \leq \alpha(2357947691) \leq 91$ \\
61917364224 & 51 & $12 \leq \alpha(61917364224) \leq 51$ \\
1792160394037 & 133 & $13 \leq \alpha(1792160394037) \leq 133$ \\
\hline
\end{tabular}

The first two powers of the prime factors 3 and $4\left(4=2^{2}\right.$, since a 2 -cycle cannot be constructed) are calculated in the table below. The number of spanning trees is equal to the power of the 3 and 4 . The number of vertices for a graph constructed of 3-cycles begins with 5 and continues to increase by a constant value of 2 . The number of vertices for a graph constructed of 4-cycles begins with 7 and continues to increase by a constant value of 3. Since the vertices for connected graphs is the sum of each sub-cycle minus the number of sub-cycles in the graph, the pattern is expected to be similar for powers of other prime numbers $(5,7,11 \ldots)$ 


\begin{tabular}{|c|c|c|c|c|c|}
\hline Exp & Powers & Prime Factorization & Spanning Trees & \# vertices & Bounds of $\alpha$ \\
\hline $3^{\wedge} 2$ & 9 & $3 * 3$ & 9 & 5 & $\alpha(9) \leq 5$ \\
\hline $3 \wedge 3$ & 27 & $3 * 3 * 3$ & 27 & 7 & $\alpha(27) \leq 7$ \\
\hline $3 \wedge 4$ & 81 & $3^{*} 3^{*} 3^{*} 3$ & 81 & 9 & $\alpha(81) \leq 9$ \\
\hline $3^{\wedge} 5$ & 243 & $3 * 3 * 3 * 3 * 3$ & 243 & 11 & $\alpha(243) \leq 11$ \\
\hline $3 \wedge 6$ & 729 & $3 * 3 * 3 * 3 * 3 * 3$ & 729 & 13 & $\alpha(729) \leq 13$ \\
\hline $4^{\wedge} 2$ & 16 & $4^{*} 4$ & 16 & 7 & $\alpha(16) \leq 7$ \\
\hline $4 \wedge 3$ & 64 & $4^{*} 4 * 4$ & 64 & 10 & $\alpha(64) \leq 10$ \\
\hline $4^{\wedge} 4$ & 256 & $4 * 4 * 4 * 4$ & 256 & 13 & $\alpha(256) \leq 13$ \\
\hline $4^{\wedge} 5$ & 1024 & $4 * 4 * 4 * 4 * 4$ & 1024 & 16 & $\alpha(1024) \leq 16$ \\
\hline $4^{\wedge} 6$ & 4096 & $4^{*} 4^{*} 4^{*} 4^{*} 4^{*} 4$ & 4096 & 19 & $\alpha(4096) \leq 19$ \\
\hline
\end{tabular}

The bounds proven by Sedlacek $\alpha(\mathrm{t}) \leq \frac{t+6}{3}$ if $\mathrm{t} \equiv 0(\bmod 3)$ and $\alpha(\mathrm{t}) \leq \frac{t+4}{3}$ if $\mathrm{t} \equiv 2(\bmod 3)$ and J. Azarija and R. Skrekovski $\alpha(\mathrm{t}) \leq \frac{t+9}{4}$ could possibly be represented by a table such as this one, although it does not make sense to plot out powers of every prime number. One thing to note about graphs constructed by connected cycles is that the bound for $\alpha(\mathrm{t})$ will be improved if the sum of the factors used for the cycle is close to each other.

Example.

For $\mathrm{C}_{36}$, the factors of 36 are $1,2,3,4,6,9,12,18,36$.

Note that:

$2 * 18=2 * 2 * 9=2 * 2 * 3 * 3=4 * 3 * 3$

$3 * 12=4 * 3 * 3$

$4 * 9=4 * 3 * 3$

$6 * 6=3 * 2 * 3 * 2=4 * 3 * 3$

For $\mathrm{C}_{36}$ and each of the graphs constructed with connected cycles, the number of spanning trees will remain the same: $\tau\left(\mathrm{C}_{36}\right)=\tau\left(\mathrm{C}_{2} * \mathrm{C}_{18}\right)=\tau\left(\mathrm{C}_{6} * \mathrm{C}_{6}\right)=36$ and so forth for each connected cycle. 


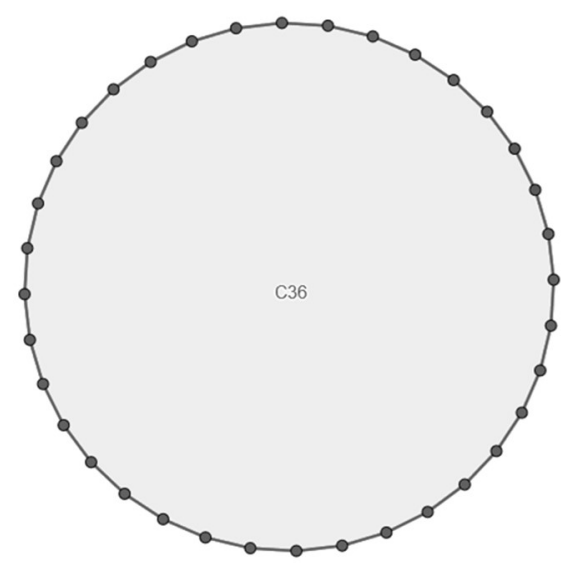

$\mathrm{C}_{36}$

$\alpha\left(\tau\left(\mathrm{C}_{36}\right)\right) \leq 36$

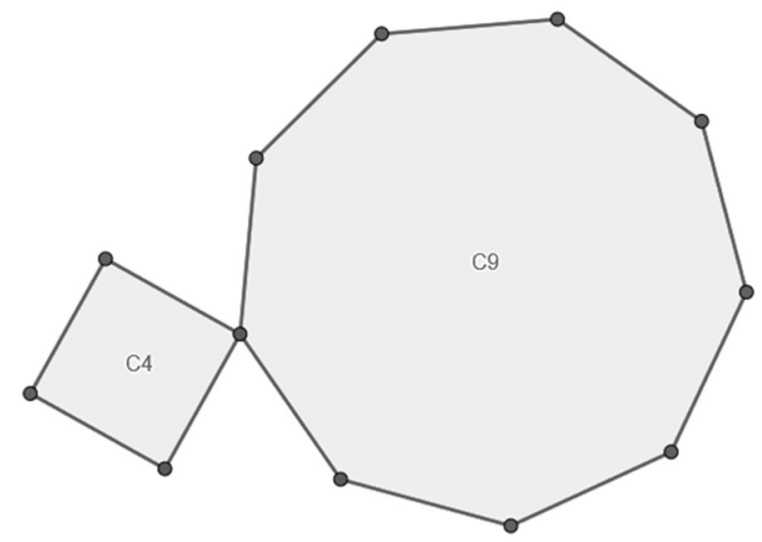

$$
\begin{gathered}
\mathrm{C}_{4} * \mathrm{C}_{9} \\
\alpha\left(\tau\left(\mathrm{C}_{4} * \mathrm{C}_{9}\right)\right) \leq 12
\end{gathered}
$$

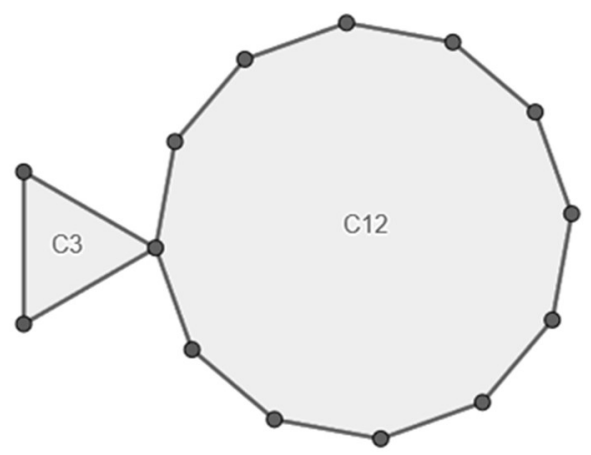

$\mathrm{C}_{3} * \mathrm{C}_{12}$

$\alpha\left(\tau\left(\mathrm{C}_{3} * \mathrm{C}_{12}\right)\right) \leq 14$

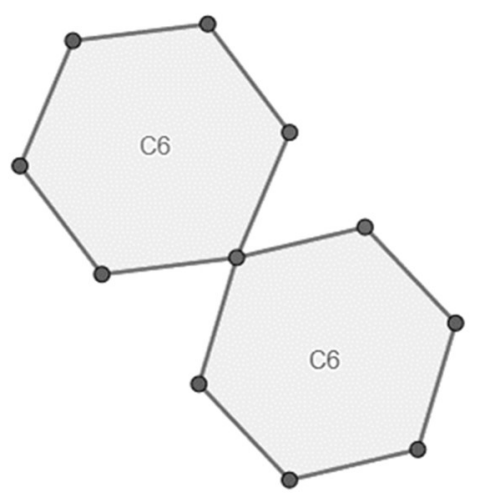

$\mathrm{C}_{6} * \mathrm{C}_{6}$

$\alpha\left(\tau\left(\mathrm{C}_{6} * \mathrm{C}_{6}\right)\right) \leq 11$

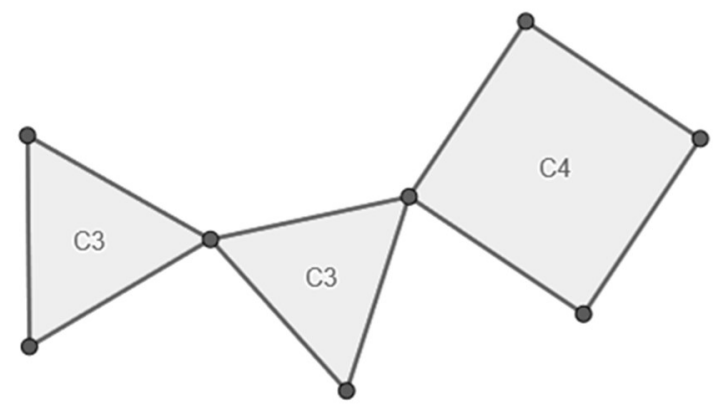

$$
\begin{gathered}
\mathrm{C}_{3} * \mathrm{C}_{4} * \mathrm{C}_{3} \\
\alpha\left(\tau\left(\mathrm{C}_{3} * \mathrm{C}_{4} * \mathrm{C}_{3}\right)\right) \leq 8
\end{gathered}
$$




\section{Conclusion}

The behavior of $\alpha(\mathrm{t})$ is observed by finding the upper bounds using properties known about $\tau\left(\mathrm{C}_{\mathrm{n}}\right)$ and $\tau\left(\mathrm{K}_{\mathrm{n}}\right)$ and improving that bound significantly by creating a graph constructed of cycles connected by one vertex. Since constructed cycles may have different factorizations, it is best to break out the cycle into multiple connected cycles that have factors that are close in number. The expected number and size of prime numbers of typical values of $\mathrm{n}$ may give us possible directions for further research. We may be able to obtain such estimates from analytic number theory. Knowing these values could offer asymptotic estimates for the number and size of cycles given in our construction. Perhaps the resulting bounds will agree with other known results. 


\section{References}

[1] 'Data Structure \& Algorithms - Spanning Tree." Tutorialspoint. Retrieved from www.tutorialspoint.com/data_structures_algorithms/spanning_tree.htm

[2] Eppstein, David, "Spanning trees and spanners", Sack, J.-R.; Urrutia, J. (eds.), Handbook of Computational Geometry, Elsevier, (1999) pp. 425-461.

[3] J. Sedlacek, On the minimal graph with a given number of spanning trees, Canad. Math. Bull. 13 (1970) 515-517.

[4] J. Azarija, R. Skrekovski, Euler's idoneal numbers and an inequality concerning minimal graphs with a prescribed number of spanning trees, IMFM preprints 49 (2011)

[5] https://mathoverflow.net/questions/93656/minimal-graphs-with-a-prescribednumber-of-spanning-trees

[6] Moore, Cristopher (2011). The nature of computation. Oxford England New York: Oxford University Press.

[7] Aigner, Martin; Ziegler, Günter M. (1998). Proofs from THE BOOK. SpringerVerlag. pp. 141-146.

[8] Weisstein, Eric W. "Circulant Graph." From MathWorld--A Wolfram Web Resource. https://mathworld.wolfram.com/CirculantGraph.html

[9] Golin M.J., Leung Y.C., Wang Y. (2004) Counting Spanning Trees and Other Structures in Non-constant-jump Circulant Graphs. In: Fleischer R., Trippen G. (eds) Algorithms and Computation. ISAAC 2004. Lecture Notes in Computer Science, vol 3341. Springer, Berlin, Heidelberg. https://doi.org/10.1007/978-3-540-30551-4_45

[10] Minimal graphs with a prescribed number of spanning trees. (2012, April 22). Retrieved from http://www.openproblemgarden.org/op/minimal_graphs_with_a_prescribed_number _of_spanning_trees 